\title{
Cuidados como categoría de análisis y orientación de las medidas socioeducativas para adolescentes en Uruguay \\ (Care as an Analysis Category and Orientation about Socio-Educational Measures for Adolescents in Uruguay)
}

OÑAT SOCIO-LEGAL SERIES VOLUME 10, ISSUE 2 (2020), 363-387: EL GÉNERO DE LOS SISTEMAS

PENALES JUVENILES: DEBATES NECESARIOS

DOI LINK: HTTPS://DOI.ORG/10.35295/OSLS.IISL/0000-0000-0000-1084

RECEIVED 27 MARCH 2019, ACCEPTED 02 OCTOBER 2019

\section{Cecilia Montes-MaldonAdo* (iD}

\section{Resumen}

El artículo aborda el problema de las medidas socioeducativas privativas de libertad destinadas a adolescentes en su tránsito por el Sistema Penal Juvenil Uruguayo. El objetivo es visibilizar cómo se compone de la ética del cuidado de agentes a cargo de la custodia de adolescentes. La investigación es de enfoque cualitativo y desarrolla un estudio de caso, único, descriptivo y en profundidad. Con enfoque etnográfico, desarrolla técnicas de entrevistas en profundidad, observación y análisis de documentos. En los resultados se pone de manifiesto el funcionamiento de la privación de libertad a través del análisis de tres elementos clave en las interacciones cotidianas en la institución: las vivencias de agentes a cargo de la custodia de adolescentes, sus interpretaciones y definiciones sobre las medidas socioeducativas y la población adolescente. Se concluye en la importancia de orientar la ejecución de las medidas socioeducativas desde el enfoque de los cuidados para mejorar las condiciones de la población adolescente en el sistema.

\section{Palabras clave}

Ética del cuidado; penalidad juvenil; prácticas estatales; Uruguay

\footnotetext{
Financiación de la Beca de Posgrado en el Exterior de la Agencia Nacional de Investigación e Innovación (ANII), Uruguay.

* Montes-Maldonado es candidata a doctora en Estudios de género: culturas, sociedades y políticas por la Universidad Autónoma de Barcelona, y el presente artículo integra su investigación desarrollada en el programa. Es licenciada en Psicología (2010) y magíster en Psicología Social (2015) por la Facultad de Psicología (UdelaR). Docente e investigadora del Instituto de Psicología Social de la Facultad de Psicología (UdelaR). Investigadora asociada del Sistema Nacional de Investigadores de la Agencia Nacional de Investigación e Innovación (ANII). Datos de contacto: Universidad Autónoma de Barcelona. Grupo de Investigación Antígona, Edificio B, Facultad de Derecho, Campus de la UAB, 08193 Bellaterra, Barcelona. Teléfono: +34 9358122 74. Dirección de email: cmontes@psico.edu.uy
} 


\section{Abstract}

The article addresses the issue of socio-educational confinement measures for adolescents in the path through the Uruguayan Juvenile Penal System. The aim is to highlight how the ethics of the care of agents in charge of the custody of adolescents is composed. The research is qualitative and develops a unique, descriptive and in-depth case study. From an ethnographic approach, it develops techniques of in-depth interviews, observation, and analysis of documents. The results show the functioning of the confinement measures through the analysis of three key elements of the institution's daily interactions: the experiences of the agents in charge of the custody of adolescents, their interpretations and definitions on socio-educational measures and the adolescent population. It concludes on the importance of guiding the implementation of socioeducational measures from the care approach to improve the adolescent's conditions in the system.

\section{Key words}

Ethics of care; juvenile penalty; state practices; Uruguay 


\section{Índice / Table of contents}

1. Introducción 366

1.1. Cuidados: la categoría omitida en el Sistema Penal Juvenil Uruguayo............. 368

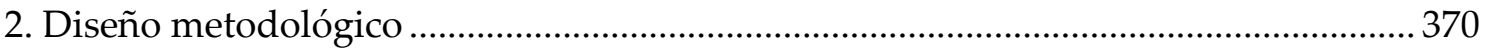

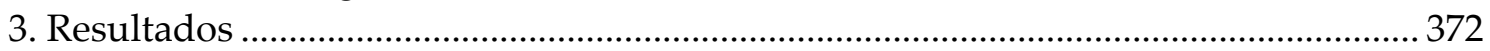

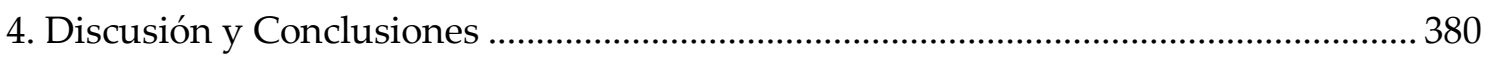

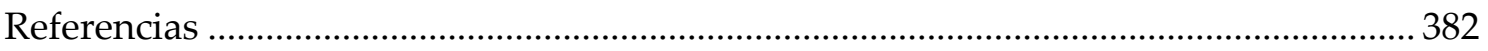

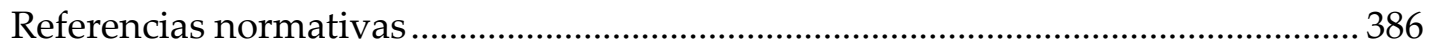




\section{Introducción}

Uruguay, concordantemente con la región y el mundo, vive un fuerte proceso de presión social que demanda ampliación del castigo por medio del endurecimiento de las penas y aumento del control hacia la población y el delito y en este caso a adolescentes y la infracción (Wacquant 2012). O, como llama Fassin (2018a), el momento punitivo, que se visualiza con el aumento de la población carcelaria en el mundo y donde el castigo que en un principio surgió como la solución al problema de la criminalidad, hoy se convierte en un problema en sí mismo.

El proceso de ampliación punitiva se expresa en diferentes aspectos y alcanza cada vez más a las poblaciones más jóvenes con propuestas que impulsan acciones de mayor control social y disminución de garantías de protección y cuidado. Si bien en 2014 no se lograron los votos necesarios en el plebiscito para bajar la edad de imputabilidad, sí se tomaron acciones que ponen en cuestión algunos de los principios de la Justicia Penal Juvenil. En este marco se aprueban instrumentos legales que marcan un retroceso normativo y contravienen las recomendaciones internacionales. Ejemplo de ello es la ley $\mathrm{N}^{\circ} 19.055$ (2013) que realiza modificaciones acortando los plazos de las sentencias en los procesos e imponiendo una pena mínima de un año de medida privativa de libertad en delitos gravísimos cometidos por adolescentes de 15-18 años.

Esta nueva ley colabora a ampliar las esferas de punición, profundizar los procesos de selectividad penal y estigmatización social de adolescentes. Profundizando de esta manera las desigualdades sociales y el castigo a las poblaciones que viven en condiciones de pobreza así como la generalización del uso de la prisión como forma de castigo primordial y legitimada (Foucault 2016).

Latinoamérica comienza un proceso de impulso de la intervención estatal hacia dispositivos de normalización de la infancia y adolescencia que despliegan procedimientos especializados y colaboran con la proliferación del modelo tutelar. Se institucionaliza la intervención estatal en temáticas de infracción a la ley y situaciones de desprotección familiar o vulneración de derechos en la primera parte del siglo XX. En Uruguay este proceso se corona con la aprobación del Código del Niño de 1934 sesgado por la doctrina de la situación irregular de la infancia que hace énfasis en la concepción de riesgo y pronóstico de peligrosidad sobre niños, niñas y adolescentes en condiciones de pobreza, inestabilidad social o provenientes de redes de cuidado precarias. Si bien hoy nuestro país cuenta con un Código de la Niñez y la Adolescencia (CNA; Ley № $17.823,2004)$ que integra los postulados de la Convención de los Derechos del Niño (Ley № 16.137, 1990) y se orienta por la Doctrina de la Protección Integral, podemos afirmar que algunas ideas y representaciones provenientes de las doctrinas tutelar y de la situación irregular de la infancia y adolescencia, coexisten. A su vez, cabe destacar la fuerte contribución que tienen estas imágenes heredadas, en la construcción y propagación de estereotipos negativos y estigmatizantes que asocian a la adolescencia con la actividad delictiva (González-Laurino y Leopold 2011, Leopold 2014).

El Sistema Penal Juvenil Uruguayo (SPJU) abarca espacios diferenciados que se corresponden con diversos sectores estatales que van desde el ámbito policial y la captación de la infracción, el ámbito de procedimiento judicial y el sector que gestiona las medidas judiciales cautelares, privativas y no privativas de libertad. Estas medidas 
son también denominadas en la legislación en la temática y en el cotidiano institucional del SPJU como medidas socioeducativas.

Todo el proceso está regido por el CNA (y diversas legislaciones posteriores que han estipulado algunas modificaciones al texto original del Código), que establece que son punibles aquellas personas entre 13 y 18 años, estando las infracciones establecidas en graves o gravísimas. Así la sanción puede implicar la medida privativa de libertad o no privativa de libertad. La legislación establece que la privación de libertad sea la última opción y por el menor lapso de tiempo posible, sin embargo en la actualidad es la medida más adoptada en los Juzgados y las medidas no privativas se utilizan en menor medida (Comité de los Derechos del Niño Uruguay 2017).

Las medidas privativas de libertad se encuentran a cargo del Instituto Nacional de Inclusión Social Adolescente (INISA), quien desde 2016 ejecutan las medidas judiciales (cautelares, privativas y no privativas de libertad) dispuestas por el Poder Judicial para la población adolescente que comete alguna infracción. Las últimas cifras publicadas por el Observatorio del INISA ${ }^{1}$ muestran la tendencia decreciente de la población que llega al sistema. Durante el año 2018 atendieron un total de 1.014 adolescentes. De la totalidad, 433 corresponden al cumplimiento de medidas no privativas de libertad (35 mujeres, 398 varones) y 588 a medidas privativas de libertad (19 mujeres, 562 varones).

Los centros donde se produce la privación de libertad son todos estatales, de carácter oficial, siendo en este momento 13 establecimientos ubicados en Montevideo (Ciudad Capital), Canelones y Lavalleja. El total de la población se distribuye entre estos centros donde son diferenciados por perfiles según características como: edad (13-15, 15-18, 18 en adelante), sexo o progresividad en el sistema. A su vez también existe un centro de ingreso, diagnóstico y derivación, un centro de máxima contención, un centro de semilibertad solo para varones y un único establecimiento para mujeres. Si bien no se analiza en el presente estudio, cabe destacar que las medidas no privativas de libertad son gestionadas por un establecimiento oficial y en ocasiones existen además convenios con Organizaciones de la Sociedad Civil (OSC).

Si bien gracias al CNA se produce un cambio radical para reconocer derechos y establecer garantías para niños, niñas y adolescentes (NNA), estudios nacionales en la temática expresan la preocupante situación de la población adolescente en lo que respecta a la Justicia Penal Juvenil y el contexto de caos y crisis permanente del sistema (González-Laurino 2015, 2016, Díaz 2019). Así como las modificaciones legislativas que colocan la situación de adolescentes que atraviesan por algún eslabón del SPJU en condiciones inadmisibles. Lo cual expresa además "una tensión entre la Doctrina de la Situación Irregular y la Doctrina de la Protección Integral, siendo la responsabilidad y la peligrosidad dos nociones que operan en simultáneo, un híbrido de paradigmas que habilita intervenciones tanto garantistas como tutelares" (López-Gallego 2017, p. 122).

En esta línea se ubican las principales áreas de conflicto y problemas que enfrenta el SPJU, identificadas por investigaciones en la temática y defensa de los derechos humanos (Comité de los Derechos del Niño Uruguay 2017, Institución Nacional de Derechos Humanos y Defensoría del Pueblo 2017, 2018, 2019, Díaz 2019) y que delimitan

${ }^{1}$ http://observatoriosocial.mides.gub.uy/portalInisa/ 
un marco de condiciones complejas para brindar un cuidado integral. Entre las principales dificultades destacan:

a. Instrumentos legales, modificaciones al CNA y funcionamiento de la Justicia Penal J uvenil contraria de la legislación internacional con fuerte aumento de la punición;

b. Alta tasa de adolescentes en el SPJU, la tercera en la región luego de Brasil y Chile con 83.6 adolescentes al 2016;

c. Excesivo uso de la privación de libertad como medida primordial y no excepcional;

d. Escasa utilización de las medidas no privativas de libertad;

e. Fallas del sistema de protección a la infancia y adolescencia; y

f. Indefinición de política pública en la temática.

Respecto al ámbito custodial-ejecución de las medidas, resaltan en los informes dificultades respecto a las condiciones de privación de libertad, las cuales son violatorias de los derechos de adolescentes; falta de definición en la ejecución de las medidas en cuanto a objetivos, procedimientos y evaluación; condiciones edilicias inaceptables en algunos centros; excesivas horas de permanencia en celdas, ocio e insuficientes propuestas educativas; falta de planificación para el egreso del sistema; uso excesivo de medicación psiquiátrica especialmente en centros con mayor horas de permanencia en celdas; recursos humanos insuficientes o con poca formación; presencia policial, uso de celdas, rejas y alambrados asimilados a penitenciarias de adultos/as.

En el artículo proponemos visibilizar algunos componentes posibles de la ética del cuidado de agentes a cargo de la custodia de adolescentes en centros destinados a la privación de libertad para adolescentes en Uruguay. Como plantea Fassin (2015) en sus análisis sobre la vida moral del Estado, las prácticas estatales pueden comprenderse a través de las personas o agentes estatales que la habitan y la hacen de forma cotidiana. Estos agentes no actúan solamente por medio de protocolos, reglas o procedimientos sino que despliegan sus afectos, valores e interpretaciones sobre la tarea y la población destinataria. La propuesta es conocer el funcionamiento de la medida privativa de libertad y cómo se compone la ética del cuidado institucional a través del análisis de tres ejes que consideramos ilustran las voces de las personas a cargo de la implementación de las medidas y las interacciones cotidianas (Medan 2017) entre agentes y población destinataria. Allí se condensan interpretaciones y definiciones que dan cuenta del contexto institucional:

a. Habitar la institución: vivencias de agentes a cargo de la custodia de adolescentes;

b. Interpretaciones sobre problema que abordan: la medida socioeducativa; y

c. La población "destinataria" de la medida socioeducativa.

\subsection{Cuidados: la categoría omitida en el Sistema Penal Juvenil Uruguayo}

La noción de cuidado (Tronto 2013) reviste una presencia controvertida en el funcionamiento de las medidas privativas de libertad. Los motivos de esta controversia son diversos y se relacionan con los enfoques teóricos y metodológicos punitivistas que históricamente se han utilizado en la implementación del encierro para los y las adolescentes que cometen infracciones (Morás 2012, González-Laurino et al. 2013, Fessler 2016, Montes-Maldonado 2019). Dichos enfoques nos conducen a la situación actual, donde la principal respuesta para la población adolescente es el encierro en condiciones 
inaceptables para sus necesidades. Por tanto, la pregunta acerca de cuáles son las acciones implementadas para cuidar o la reflexión en torno a las capacidades de cuidado del sistema hacia la población que atienden, se encuentra en falta u omitida.

Nos interesa pensar la noción de cuidados desde una perspectiva amplia e integral, que trasciende las individualidades, el ámbito de lo privado y que integra una multiplicidad de dimensiones conectadas entre sí. Desde la definición de Fisher y Tronto son aquellas actividades que realizamos las personas como parte de un proceso vital que se va transformando en función de las diferentes etapas. Las actividades de cuidado se comprenden en relación con su contexto de producción político y cultural, cuidar es una práctica compleja enmarcada en relaciones de poder y conlleva conflicto.

... una actividad humana que incluye todo lo que hacemos con vista a mantener, continuar o reparar nuestro 'mundo', de tal manera que podamos vivir en él lo mejor posible. Este mundo incluye nuestros cuerpos, nuestras individualidades y nuestro entorno, todo lo que buscamos para tejer una red compleja que sostiene la vida. (Tronto 1993, p. 103)

Tronto $(1993,2013)$ colabora a su vez en la ampliación de la discusión sobre la ética de los cuidados introducida por Gilligan (1982). Así, coloca en primer plano las vivencias, voces y moralidades de las personas encargadas del cuidado de otras personas, de la necesidad de reconocer las vulnerabilidades, apreciar el trabajo de cuidados como algo sustancial en el sostenimiento de la vida y reconocer que circulamos por relaciones de cuidado y nuestra condición de personas interdependientes. En su definición destaca cuatro elementos centrales o cualidades morales necesarias para comprender la ética del cuidado: atención, responsabilidad, competencia y sensibilidad. Y recientemente agrega un quinto elemento que lo llama solidaridad o confianza.

Estas ideas se contraponen a la tradicional visión de las mujeres como aquellas más capacitadas para cuidar o únicas responsables en la tarea. Estos aportes abren el debate sobre la democratización del cuidado, la construcción de vínculos de corresponsabilidad y la ruptura de las relaciones de género en base a los roles tradicionales para apostar a generar condiciones de vidas más vivibles (Butler 2010).

Desde este marco de discusiones postulamos la necesidad de problematizar la noción de cuidado en la actual gestión de la privación de libertad de adolescentes en Uruguay. En este contexto la gestión del cuidado se realiza en condiciones particulares que debemos tener presentes para su análisis: el ámbito de ejecución del cuidado es institucional y normativo; el lugar es de encierro; las personas encargadas de cuidar son contratadas para la tarea. Estas particularidades ponen en tensión las clásicas discusiones sobre el cuidado que se producen tradicionalmente relacionadas con el ámbito de la familia y enmarcadas en relaciones de afecto. Este contexto singular, hace necesaria la aclaración de porqué es importante conocer cómo significan el cuidado quienes se encuentran a cargo de la privación de libertad de adolescentes, qué prácticas de cuidado identifican, impulsan y cómo se va componiendo la ética del cuidado en la institución.

Transversalizar el concepto de cuidados es una herramienta útil de análisis para problematizar la forma en que se implementan las medidas privativas de libertad para adolescentes. Reviste relevancia la posibilidad de debatir y cuestionar el tipo de atención que reciben en estos espacios de encierro, cuáles son las condiciones en las que se 
encuentran y cuáles son los discursos en torno al cuidado que circulan entre los y las referentes institucionales.

Posicionar la investigación desde los debates del cuidado implica destacar su aporte desde diferentes aspectos y plantear que el eje orientador del tratamiento de adolescentes en el sistema debe ser el de colocar al cuidado en el centro.

Como primer aspecto destacamos que el cuidado es un derecho humano universal y fundamental (Tronto 2005, Pautassi 2010). Si bien la comisión de una infracción ingresa a la población adolescente al mapa de las políticas punitivas y de seguridad, la implementación de las mismas también debe garantizar el cuidado y respeto de todos los derechos humanos. Cabe destacar que la legislación actual establece que el cumplimiento de la medida judicial de privación de libertad debe además reconocer y garantizar los derechos establecidos en la legislación nacional e internacional vigente (Convención sobre los Derechos del Niño 1989, Ley nº 17.823, Ley N 19.367).

El segundo aspecto, es situar el potencial de los cuidados como categoría de análisis social (Faur 2014) y de análisis de los sistemas de bienestar o social care (Daly y Lewis 2000). Su potencial analítico se ve profundizado en su intersección (Crenshaw 2004) con las dimensiones de desigualdad social, género, raza y clase social, principalmente.

Y tercero destacamos la potencialidad de articular género y cuidados para el análisis del problema planteado. El concepto de cuidados tiene un gran impulso en el marco de diversas disciplinas que discuten sobre el cuidado como trabajo en los años sesenta y setenta del siglo XX. Luego los estudios de género han ido ampliando las discusiones desde múltiples ópticas y el movimiento feminista se ha encargado de darle impulso y visibilidad a la discusión (Carrasco et al. 2011). Además la posibilidad de analizar las relaciones de género y las configuraciones que se establecen para el funcionamiento de la privación de libertad, los ideales de cuidado (Hochschild 2011) imperantes, son un aporte fundamental para comprender las prácticas de cuidado que se realizan y analizar su dimensión moral (Fassin 2015) o componentes morales (Vianna 2010).

\section{Diseño metodológico}

La investigación cualitativa que aquí se presenta cuenta con un corpus que abarca actividades desarrolladas en dos espacios institucionales, Instituto del Niño y Adolescente del Uruguay (INAU) e INISA. En cuanto a las técnicas empleadas, una totalidad de 34 entrevistas en profundidad, sistematización de 12 legajos y seis historias de vida (denominación institucional del archivo individual de cada NNA), observaciones y registro en diario de campo, han ofrecido abundante información acerca de los contextos de encierro de adolescentes. A través de la estrategia de triangulación de técnicas (Ruiz Olabuénaga 2012) garantizamos la validez y calidad del estudio por medio del uso de diferentes fuentes de información.

Para los objetivos del presente artículo se utilizan únicamente los materiales producidos en INISA que abarca 15 entrevistas en profundidad a personal-agentes que realiza tareas vinculadas de forma directa a cargo de adolescentes, 12 legajos de adolescentes y registro de observaciones en diario de campo.

De esta manera, el estudio integra las metodologías cualitativas (Denzin y Lincoln 2018) de la investigación social con perspectiva etnográfica (Hammersley y Atkinson 1994, 
Guber 2013). El itinerario planteado para alcanzar los objetivos dispone un estudio de caso único, descriptivo (Yin 1993) y en profundidad (Flyvbejereg 2004).

Por su parte, los aportes de las epistemologías feministas (Haraway 1995, Harding 2006) son una guía fundamental como perspectiva y para delimitar la estrategia de posicionamiento en el campo, con los y las participantes de la investigación y para el análisis de los procesos de reflexividad (López-Gallego 2014) que atraviesan el estudio.

El contexto institucional donde se producen los datos se caracteriza por ser un espacio de encierro y aislamiento -centros de privación de libertad para adolescentes-, institución total (Goffman 2001) gestionada por un ente estatal y enmarcado en circuitos de administración burocráticos Estos elementos componen un escenario diverso y complejo donde el acceso y la permanencia en la institución configuran un desafío. El trabajo de campo abarca los meses de septiembre a diciembre de 2017 en Montevideo, Uruguay.

Para el análisis de la información producida seguimos la propuesta de análisis temático cualitativo (Spencer et al. 2014). Con la ayuda del software de análisis de datos cualitativos asistido (CAQDAS) MaxQda versión-2018, se llevan adelante las cinco fases de la propuesta de análisis. Siendo estas; familiarización con la totalidad de los datos; elaboración del marco temático inicial; indexación y clasificación de los datos; relectura de los segmentas clasificados y enriquecimiento del marco temático. Y por último la síntesis y visualización de los datos.

Cabe destacar que la investigación cuenta con el aval del Comité de Ética de Facultad de Psicología (Universidad de la República) así como las autorizaciones pertinentes de las instituciones públicas nombradas. El procedimiento ético sigue las sugerencias de la American Psychological Association (2017) en cuanto al cuidado de confidencialidad, privacidad y anonimato de las personas participantes y el uso del consentimiento informado firmado que incluye la voluntariedad de participación.

A continuación se presenta de manera resumida el corpus de la investigación donde se detallan las técnicas empleadas, sexo y rol en la institución de las personas participantes. Así como también los diferentes tipos de Centros donde se desempeñan. 
TABLA 1

\begin{tabular}{|c|c|c|c|c|}
\hline \multicolumn{2}{|c|}{$\begin{array}{l}\text { Participantes de } \\
\text { entrevistas en profundidad }\end{array}$} & \multicolumn{2}{|c|}{ Legajos de adolescentes* } & \multirow{2}{*}{$\begin{array}{c}\text { Observación } \\
\text { Tipos de Centros: }\end{array}$} \\
\hline Total: & 15 & Total: & 12 & \\
\hline Varones & 6 & Varones & 10 & Mujeres \\
\hline Mujeres & 7 & Mujeres & 2 & Varones \\
\hline \multicolumn{2}{|l|}{ Roles por área: } & \multicolumn{2}{|c|}{ Contenido legajos: } & $\begin{array}{l}\text { Varones de } 13 \text { a } 15 \\
\text { años }\end{array}$ \\
\hline Educador/a o instructor/a & 3 & \multirow{2}{*}{\multicolumn{2}{|c|}{ Formularios de Ingreso y datos básicos }} & $\begin{array}{l}\text { Centro de Máxima } \\
\text { Contención }\end{array}$ \\
\hline $\begin{array}{l}\text { Área Psi**: } \\
\text { 5-Psicología y } \\
\text { 1-Psiquiatría }\end{array}$ & 6 & & & $\begin{array}{l}\text { Centro de } \\
\text { Cumplimiento de } \\
\text { Medida en semi- } \\
\text { libertad } \\
\end{array}$ \\
\hline Educación Social & 1 & \multirow{2}{*}{\multicolumn{2}{|c|}{$\begin{array}{l}\text { Registros de intervención realizada: INISA y otras } \\
\text { instituciones (derivantes, en coordinación) }\end{array}$}} & \begin{tabular}{|l|} 
Programa de \\
Inserción Social y \\
Comunitaria \\
\end{tabular} \\
\hline Trabajo Social & 3 & & & $\begin{array}{l}\text { Programa de } \\
\text { asistencia luego } \\
\text { del egreso } \\
\end{array}$ \\
\hline Área J urídica** & 1 & \multirow{2}{*}{\multicolumn{2}{|c|}{$\begin{array}{l}\text { Documentación personal: } \\
\text { Cédula de identidad, partidas de nacimiento, } \\
\text { constancias de instituciones educativas, } \\
\text { intervenciones médicas, otros/as }\end{array}$}} & \\
\hline Dirección/Coordinación & 1 & & & \\
\hline & & \multicolumn{2}{|c|}{$\begin{array}{l}\text { Documentación de funcionamiento interno: } \\
\text { autorizaciones de visitas, constancias médicas por } \\
\text { revisaciones ante agresiones o peleas, licencias } \\
\text { solicitadas o concedidas, informes de ingreso y } \\
\text { egreso de centros, constancias de traslados de } \\
\text { centros }\end{array}$} & \\
\hline & & \multicolumn{2}{|c|}{$\begin{array}{l}\text { Documentación Judicial: registro de audiencias, } \\
\text { resoluciones judiciales, oficios judiciales, } \\
\text { autorizaciones de salidas }\end{array}$} & \\
\hline
\end{tabular}

Tabla 1. Corpus de la investigación: INISA.

(Fuente: elaboración propia con datos de 2017.)

\section{Resultados}

Exponemos aquí un análisis sobre el funcionamiento de las medidas privativas de libertad en el contexto de los centros destinados para este fin en Uruguay. Seguimos el objetivo de visibilizar y problematizar la composición de la ética del cuidado imperante en este contexto institucional por parte de agentes a cargo de la custodia de adolescentes. Con este objetivo ahondamos el análisis en tres puntos que consideramos clave dado que condensan los elementos más controvertidos a la hora de implementar la medida socioeducativa de privación de libertad.

Partimos de la premisa que las prácticas que rodean la gestión de la privación de libertad atraviesan procesos de interpretación de las legalidades, normas y protocolos que los regulan y en función de esas interpretaciones van definiendo la tarea y construyendo las relaciones. Relaciones estas, que no son permanentes ni estables y que se van 
transformando entre las personas que trabajan en la institución y la población destinataria de las medidas socioeducativas (Fassin 2015).

En este sentido valoramos el carácter performativo (Butler 1993) de la ejecución de las medidas socioeducativas dado que se van construyendo por, repetición, perdurabilidad en el tiempo y a través de procedimientos rituales. Situamos así estas tres líneas analíticas en función de su potencial para pensar las tensiones sobre las posibilidades limitadas de cuidar en un espacio de encierro y aislamiento para adolescentes.

\section{a. Habitar la institución: vivencias de agentes a cargo de la custodia de adolescentes}

La actual gestión de las medidas privativas de libertad se halla a cargo del INISA desde 2016. Momento en el que se crea esta nueva institucionalidad luego de la histórica atención en esta área realizada por el Instituto del Niño y Adolescente del Uruguay (INAU). La historia institucional es larga y compleja excediendo los objetivos del presente artículo. Sin embargo, mostrar algunos efectos de la misma de la mano de los relatos del personal que se ocupa diariamente de la gestión de los centros de privación de libertad es una forma de acercarnos a describir la vida institucional, desde las vivencias y afectos de las personas que la habitan. Cabe destacar que este habitar es diverso, múltiple y proviene de la experiencia de cada persona, las interpretaciones y significados del rol que les toca ocupar en la institución. Las interacciones cotidianas en la institución son variadas y entre diferentes agentes que pueden transitar por allí como ser: adolescentes, familias y/o referentes, equipo del centro, cargos de coordinación y dirección del centro, personal de otras áreas de la institución, Poder Judicial, referentes de instituciones con las cuales se realizan coordinaciones o derivaciones.

Consideramos que la trayectoria institucional y las formas de significar las vivencias del personal de los centros tiene incidencia en la forma en que desempeñan su tarea y fundamentalmente en las relaciones que establecen con los/as adolescentes.

Pensamos en concordancia con Goffman (2001) que los centros de privación de libertad, como espacios de encierro, constituyen instituciones totales. Las formas de organización de la institución genera que las personas desarrollen todas las actividades que hacen a la gestión de su vida, estando las variables temporo-espaciales distribuidas desde un afuera-la institución y sus responsables- quiénes establecen de forma homogénea las necesidades y formas de resolverlas y realizan tareas de control y vigilancia. De esta manera se configuran dos grupos distintos, por un lado, la población que cumple con la medida judicial y por otro, el personal del centro encargado de hacer cumplir esa medida. En la institución total los roles y posiciones asimétricos se diferencian, colaboran en mantener la distancia de las posiciones en la organización social, el estatus que a su vez redunda en la diferencia entre personal y adolescentes. Además se establece la escisión adentro-afuera bien marcada que en el caso de la privación de libertad separa y genera aislamiento entre adolescentes y sus lazos familiares, sociales y comunitarios.

Goffman (2001) sostiene que en el trabajo cotidiano con seres humanos se produce el desarrollo de un clima moral, así como Fassin $(2015,2018 b)$ habla de la producción de subjetividades morales para explicar que en el día a día las prácticas de los equipos están transversalizadas por sus ideales y sentimientos y por ende conocerlos nos permite analizar la institución. Es interesante así detenerse en las valoraciones que el propio personal hace de sí mismos y del funcionamiento institucional. Las citas de entrevistas 
muestran las sensaciones de disconformidad, falta de información y lineamientos claros en dos aspectos. Por una parte, a nivel de la política institucional macro y por otra, de la planificación de trabajo, objetivos y forma de funcionamiento de cada centro, a nivel más micro. Este malestar es un efecto de la crisis permanente antes nombrada, y de que en los últimos años la institución ha cambiado varias veces de formas de conducción, nombre, autoridades principales y por último la creación de una nueva institucionalidad que se desliga de la anterior pero que aún se encuentran en transición en algunas áreas.

Respecto a la situación de cambio constante de autoridades, un Educador con muchos años de trabajo en la institución plantea.

A ver, no es una política de Estado que se pudiera encarar independientemente del gobierno que hubiera. Cada gobierno le daba su perfil. Y se lo daba a través de las personas que ocupaban los cargos de dirección y gestión. Entonces parecía, mirá, parecían ciclos que se instalaban en el Directorio. Había que esperar, nosotros teníamos desde el llano, que esperar a que esa gente se instalara, empezara a conocer, designar mandos medios, etcétera. Y bueno, después de eso había un período de tiempo que se implementaban algunas cosas, que no eran demasiadas tampoco, sino que se trataba de mantener lo que había, siempre hubo una institución o esta parte de la institución que parece que es un reflejo de la población con la que trabajamos. O sea, la marginalidad. Mirá esto, ¿Ves esto? [Se refiere a las condiciones edilicias. Señala paredes rotas, con humedad]. Nosotros cuando vinimos acá pusimos el grito en el cielo porque no era posible. (E1-Hombre-Educador)

Por su parte, las citas de entrevistas de una mujer del área psi y una Educadora dan cuenta de los efectos de falta de conducción y de situación general de crisis que genera efectos además en la salud y el desempeño del personal.

No te lo va a decir nadie, todo el mundo se está cuidando, yo entré por concurso, tengo la libertad, la dignidad y la honestidad para poder decirlo. Entonces, no quiere decir que todos sean malos, hay gente muy buena y con muchas ganas pero hay una mole que aplasta, aplasta y enferma. Hay muchas licencias y la gente que realmente tiene ganas termina aplastada en esta máquina de picar carne. (E7-Mujer-Área Psi)

... para mí es un desastre muchas cosas del programa, como se implementaron, porque hay un abandono institucional, tenemos un año trabajando sin objetivos de programa, sin planificación, autogestionándonos, con una dirección que no era dirección (...) es como re perverso, estuvimos como en un conflicto y entonces la tenía como en el aire a una dirección que no hacía más que hostigarnos, y sin objetivos, entonces ahí... Nosotros pudimos hacer un año de plancha ['Hacer la plancha' significa hacer lo mínimo por el trabajo]. (E9-Mujer-Educadora)

Este contexto provoca la situación de inestabilidad que muestran las citas de entrevistas y también deja de manifiesto cierta forma de funcionamiento que se relaciona con lo que Haney (2004) denomina gobierno por contradicción, a través del despliegue de diversos mensajes y formas de actuar, que unas veces pueden ser ambiguos y otras contradictorios. Estas incoherencias en el accionar afectan a la totalidad del colectivo institucional, adolescentes y agentes que se desempeña allí y también deja entrever cómo las instituciones totales tienden a descentrarse de los objetivos explicitados formalmente, en función de otros objetivos que pueden ser estratégicos o utilizados para justificar algunas decisiones o procedimientos (Goffman 2001). Sigue a este análisis la cuestión de 
las interpretaciones sobre cómo implementar la medida socioeducativa y sus imprecisiones.

\section{b. Interpretaciones sobre el problema que abordan: la medida socioeducativa}

Llamamos problema aquí al objetivo institucional de INISA, el foco de trabajo que tiene entre manos el equipo de los centros de privación de libertad, es decir, implementar la medida judicial de privación de libertad o medida socioeducativa dictaminada por el Poder Judicial y realizar la custodia de la población adolescente. De la legislación no surgen directivas o instrucciones claras de cómo debiera ser la gestión de la privación de libertad o la forma que reviste el cumplimiento de la medida socioeducativa, sino que establece algunos lineamientos muy generales que pueden y son interpretados de diversas maneras por los equipos de los centros.

Por un lado, nos encontramos con las disposiciones del CNA en los capítulos IX y X, que delimitan el procedimiento en cuanto a adolescentes y la infracción a la ley penal. Respecto a la privación de libertad se establece que “El régimen de privación de libertad consiste en recluir al adolescente en un establecimiento que asegure su permanencia en el recinto, sin menoscabo de los derechos consagrados en este Código, las normas constitucionales, legales e instrumentos internacionales" (Ley n⿳0 17.823, Código de la Niñez y la Adolescencia).

Y, por otro lado, encontramos, la delimitación de objetivos institucionales del organismo encargado de implementar las medidas judiciales privativas y no privativas de libertad, INISA:

El Instituto Nacional de Inclusión Social Adolescente tendrá como objetivo esencial la inserción social y comunitaria de los adolescentes en conflicto con la ley penal mediante un proceso psicosocial, educativo e integral, que conlleve el reconocimiento de su condición de sujetos de derecho. (Ley $\mathrm{N}^{\circ}$ 19.367)

La privación de libertad es el mecanismo más utilizado como forma de sanción cuando se hallan adolescentes que cometen alguna infracción (Comité de los Derechos del Niño Uruguay 2017). Como plantea Fassin (2018a) alrededor del acto de castigar encontramos múltiples discursos y disciplinas que han edificado el campo de saberes del castigo. Por ese motivo comprender cómo las personas encargadas de los centros definen, justifican y distribuyen el castigo es de interés analítico para entender las relaciones entre crimen y castigo, entre las disposiciones normativas establecidas y las prácticas burocráticas concretas.

Así, consideramos relevante el análisis de estos aspectos dado que las formas de entender los objetivos de la tarea, la metodología u enfoques y el rol en la institución, va dibujando las relaciones e interacciones cotidianas (Medan 2017) que se establecen entre el personal de los centros y adolescentes. Los objetivos establecidos se pueden ver influidos por la formación disciplinaria, aspectos biográficos y de historia en la institución, el tipo de centro donde se desempeña y el proyecto de centro $u$ otros elementos vinculados a sus concepciones de transgresión, adolescencia, entre otros. De esta manera las personas entrevistadas interpretan y argumentan el problema y objetivo sobre el cual trabajan abarcando diferentes fines, como ser: rehabilitar, responsabilizar, educar o reeducar, encerrar. 
En este sentido un Educador reflexiona alrededor de lo que denomina el deber institucional de la responsabilidad.

A ver, el deber institucional, es el tema de la responsabilidad de lo hecho. Ellos tienden acá a entrar y como que lo que hicieron ya pasó y fue olvidado. Desde la rapiña hasta un homicidio; pasó, fue, murió alguien... Ya ni fantasmas, aunque muchos conviven con eso no muy bien. (E1-Hombre-Educador)

En esta línea ilustramos además en palabras de un Trabajador Social que se pregunta por el cuidado en forma de contraste con el cumplimiento de la medida.

¿Cómo esto del cuidar a los adolescentes?, porque bueno la institución tiene como este fin que es más bien responsabilizar, ¿no? El hacer cumplir una medida a partir de una infracción o falta. (E11-Hombre-Trabajo Social)

Responsabilidad, responsabilizar, responsabilización son nociones que se repiten en el cotidiano institucional a partir de los discursos de agentes institucionales y también se visualizan en la producción escrita: informes de seguimiento al Poder Judicial $u$ otros documentos. Sin embargo, los usos de estos términos son difusos y ambivalentes, muestran los sesgos de discursos disciplinares expertos y además tienen una clara incidencia en los procesos judiciales de adolescentes. Investigaciones realizadas centradas en comprender la noción de responsabilidad (López-Gallego y Padilla 2013, González-Laurino y Leopold 2013), nos alertan sobre los diversos usos de esta noción en torno de las medidas socioeducativas. Por un lado, destacan que la responsabilidad como concepto jurídico comienza a utilizarse como noción clave en el proceso de cambio de paradigma hacia la protección integral de la población adolescente en la justicia juvenil y que tiene en cuenta la situación especial de, crecimiento, desarrollo y autonomía progresiva. Pero en su uso desde las prácticas cotidianas concretas, la misma ha estado relacionada con las exigencias de asunción de culpabilidad, arrepentimiento y confesión. A partir del análisis de expedientes observan el lugar en que queda la población adolescente en el trayecto de las medidas socioeducativas, constreñida a "producir un relato biográfico que muestre que comprenden la situación en la que se encuentran, reflexionen críticamente sobre los actos infraccionales cometidos y proporcionen pruebas de los esfuerzos que realizan para asumirse responsables y superarse a sí mismos" (González-Laurino y Leopold 2017, 74).

Es interesante además mostrar el análisis de Uriarte (2013) al respecto, quien plantea que la responsabilidad tiene en su espíritu el énfasis en poner garantías a los intentos de intervención puramente punitiva. A su vez muestra como a partir de una interpretación del Artículo 79 del CNA (2004) la responsabilidad se empezó a mirar como el contenido de la medida socioeducativa y advierte que uno de los intentos del poder punitivo es convertir la asunción de responsabilidad en confesionarios para adolescentes.

El siguiente extracto de entrevista deja planteada las dificultades estructurales y de recursos de la institución para la ejecución de las medidas. Así como la forma en que se va colocando la responsabilidad o las posibilidades de restitución de derechos para adolescentes.

Estamos ante una estructura como pudiste ver acá, que mucho no rehabilita ¿no? Digo, no es porque la gente no lo desee sino porque bueno, es lo que hay. Digo, si tú conoces 
o pudiste ir a la Colonia Berro, ${ }^{2}$ las edificaciones son de hace 150 años, es decir, son lugares que no se adaptaron a tener gente de repente privada de libertad, o gurises [adolescentes] privados de libertad, pero no sería lo ideal. Yo creo que ese es el gran debe de la institución. No solo para ellos, sino también para quienes trabajamos ahí. (E4-Hombre-Área Psi)

En el tránsito cotidiano por la institución se puede visualizar la preponderancia de la tendencia a colocar la responsabilidad de la infracción en la individualidad de adolescentes o sus familias. Y la voluntad de salir de esta situación en capacidades también individuales a través del esfuerzo o perseverancia que deberían tener. Esta tendencia se halla alejada de las perspectivas que muestran que algunas explicaciones de las infracciones o delitos tienen fundamentos además desde lo social, el Estado y sus instituciones. Asimismo, interesa destacar que las sanciones o castigos siempre van más allá de la persona encerrada, alcanzando a sus familias y comunidades como poblaciones punibles, según Fassin donde cierta distribución del castigo contribuye a profundizar las desigualdades sociales en las poblaciones que ya se encuentran desfavorecidas (Fassin 2018a).

Esta tendencia es muestra además de las políticas de individuación (Merklen 2013) como nuevas formas de control social donde se exaltan las nociones de responsabilización o reactivación individual. Las mismas, dan cuenta del traspaso de las dificultades socioeconómicas, desigualdades sociales y posibles riesgos hacia las individualidades. Así como también de la precarización de las protecciones sociales existentes. En palabras de Merklen, “'Responsabilización' y 'Reactivación' de la voluntad individual constituyen mecanismos discursivos, articulados en el seno de una verdadera ideología al servicio de la instauración de políticas de la individuación" (Merklen 2013, 48).

¿De qué maneras se pueden frenar los intentos de proponer intervenciones cada vez más punitivas? En un contexto donde desde todos los ámbitos sociales se pide más encarcelamiento y se avalan condiciones inaceptables para los/as adolescentes, pareciera una pregunta difícil de contestar.

En esta línea, se puede ver nuevamente cómo afecta la falta de definición política y de una estrategia de ejecución de las medidas judiciales y las perspectivas que se siguen. Una Educadora reflexiona en este sentido y muestra las dificultades de los equipos de trabajo generados a partir de los constantes cambios en las directrices, los imprevisibles y la poca participación que tienen en las transformaciones.

[las medidas] Es que no las ejecutan, las dejan a la suerte de las personas que estén, para mí no hay una política institucional. Más allá de lo discursivo para mí no hay. Ósea porque es claro porque no podés tener tantos centros, tá, que eso también es una discusión, hasta qué punto eso no es una forma de ejecutar; pero para mí no las ejecutan, es como que las dejan a la suerte de las personas (...). Te das cuenta de que no hay una política porque si no, no puede cambiar tanto. Vos nunca sabés que es lo que va a pasar acá, nunca sabés qué va a pasar con un centro. El año pasado fue absurdo, de un día para el otro un centro cerraba y abría en otro lugar. (E9-Mujer-Educadora)

En el marco de la proliferación discursos punitivistas y la sensación de abandono institucional que destacan las personas entrevistadas nos preguntamos por el enclave

${ }^{2}$ Colonia Berro: gran predio en el Departamento de Canelones (Uruguay) donde se encuentran varios de los Centros de Privación de Libertad. 
educativo de la medida socioeducativa. En las entrevistas, la cuestión educativa aparece asociada a la educación como un derecho, educación formal o no formal o lo educativo en tanto proceso de construcción de ciudadanía o crecimiento personal.

Entonces bueno, esa medida implica que ahí nosotros tenemos que hacer todo; un diagnóstico de la situación del joven, armar un proyecto de trabajo y bueno. Y las medidas han cambiado obviamente, han cambiado en tiempo, en duración. Y si me parece que también depende de los centros, cada centro tiene proyectos distintos también, entonces eso también. Acá la prioridad del centro es el tema de lo educativo, los chiquilines [adolescentes] todos tienen que estudiar sea con la maestra si no terminaron la escuela, o el liceo; talleres, acá adentro hay muchos talleres. Entonces bueno, ocupar ese tiempo, volver al tema del estudio que la deserción del tema del estudio es importante, volver a motivarse con el tema del estudio, valorar el tema del estudio. Entonces digo, depende de cada centro el tema de cómo se instrumenta la medida socioeducativa. (E13-Mujer-Trabajo Social)

Yo creo que, al ser una medida judicial, tenés que cumplirla. Y la medida se cumple. Se trata de ser muy rígido en el cumplimiento ese. Pero obviamente que para los que entendemos que, si te dan a un adolescente para que esté acá, es para que hagas algo más que cumplir una medida. Tiene que haber algo más. También trabajo educativo para que la educación formal sea lo más que pueda, de hecho, hay jóvenes que terminaron Ciclo Básico, que aprueban Ciclo Básico, que terminan Primaria. (E12Mujer-Educación Social)

Podemos observar nuevamente la dispersión de las formas de llevar adelante la medida socioeducativa y cómo estás formas dependen de la conducción de cada centro y lo que allí se priorice.

Un estudio reciente (Ruiz Barbot et al. 2018) que ha censado a 265 adolescentes privados de libertad (de un total de 346 adolescentes) da cuenta de que solamente un 3\% de la totalidad contaba con un nivel educativo a nivel de educación formal esperado respecto a su edad, y solo un 5\% tenía aprobado el Ciclo Básico de Educación Secundaria. A su vez muestran entre los resultados, que las trayectorias educativas están basadas en el fracaso, los procesos discontinuos o la desvinculación del sistema.

\section{c. La población "destinataria" de la medida socioeducativa}

En la institución y los centros circulan diversas definiciones o caracterizaciones de cómo son, cómo se comportan y qué pronóstico puede tener la población de adolescentes que ingresa. Estas características, también denominadas perfiles abarcan tanto a adolescentes como a su contexto y espacios de socialización más próximos: familias y referentes significativos y contexto territorial: barrios, comunidades, otras instituciones a las cuáles se vincularon en su historia.

Se puede pensar a través de los relatos de las entrevistas en cómo se van elaborando determinados esquemas interpretativos (Goffman 2001) en la institución total por medio del cual se erige una concepción e interpretación homogénea de cómo son y se comportan los/as adolescentes. De alguna manera esta forma de ubicar a la población con la que trabajan dentro de determinadas categorías colabora en la construcción de un/a otro/a como lejano y diferente.

Muestra de la construcción del otro/a como ajeno/a es esta cita de una entrevista con un Educador. 
... viven en mundos paralelos, submundos que se manejan de otra manera. Existe hasta una Justicia de manejo propio. Yo resuelvo mis problemas a mi manera. (E1-HombreEducador)

A su vez ciertas características que establecen los equipos de los centros, van en la línea de la cimentación del estigma (Goffman 1963) que rodea a la población adolescente que comete infracciones. Los estigmas refieren a aspectos desvalorizados que se utilizan para desacreditar con sus concomitantes de producción de inferioridad y potencial peligro. $\mathrm{O}$ como expresa Rodríguez-Alzueta que los jóvenes pasan por un triple proceso de estigmatización donde "resultan estigmatizados por los vecinos, y los medios masivos de comunicación, sobreestigmatizados por los policías y empapelados por las agencias judiciales" (Rodríguez-Alzueta 2016, 26).

Así surgen abundantes afirmaciones generalizantes que asocian infracción $y$ adolescencia con violencia, uso de drogas, vagancia, vida en los márgenes de las instituciones y prácticas catalogadas como carcelarias. Elementos estos que colaboran en la desacreditación de los y las adolescentes como se evidencia en estas citas.

Los adolescentes tienen severos problemas familiares, familias disfuncionales, padres presos, drogas, padres ausentes, familias monoparentales, no conocen al padre nunca, o todos delinquen. (E14-Mujer-Área Psi)

Así como también aparece la tendencia a idealizar el pasado intentando mostrar que la situación ha ido empeorando.

Ha habido un cambio en las causas también, y se observa eso, un cambio también. Si bien antes capaz que ingresaban chiquilines [adolescentes] por hurto, ahora ya ingresan con situaciones más complejas, rapiñas más complejas, la droga y todo el círculo que rodea todo el tema de la droga, el consumo de droga, venta, todo eso lo que implica; y después homicidios también; después todo el tema de delitos sexuales también. (E12Mujer-Trabajo Social)

Cuando se relaciona y reflexiona sobre adolescentes con sus vínculos más próximos ya sean familiares biológicos $\mathrm{u}$ otros referentes afectivos, en general son asociados con elementos negativos. La noción de familia más utilizada sigue los patrones normativos en base a una determinada configuración -moderna, nuclear, estable- que se aleja de la realidad de parte de esta población. Quedando así las configuraciones alternativas a la valorada positivamente por los equipos técnicos, en posiciones de amenaza, desconfianza o sospecha.

Las valoraciones morales también se cuelan hacia los entornos de adolescentes considerando desde determinados ideales de cuidado (Hochschild 2011) aquellas prácticas de crianza que se valoran como buenas o malas.

Pero siempre son de contexto vulnerable, el tema de las drogas también está bastante atravesado con la población y bueno, a nivel de referentes familiares siempre son también familias desestructuradas en cuanto a que por lo menos siempre falta uno de los referentes, casi siempre es el padre, pero... te diría que por lo menos en el noventa por ciento, falta uno de los dos, en muchos los dos, o que han estado privados de libertad, entonces siempre hay alguien más, se ha criado con... como unos vínculos muy itinerantes. (E8-Hombre-Área Psi) 
... el 99,0\% de los chiquilines [adolescentes] de los que están acá vienen de familias disfuncionales. O sea, o no vienen de familia mucho de ellos. O muchos de ellos vienen del INAU. (E5-Mujer-Área Jurídica)

Un elemento también destacado es el lugar geográfico de donde provienen o donde han residido. El censo realizado por Morás (2016) sobre 522 adolescentes que se encontraban en privación de libertad muestra que para adolescentes de Montevideo (283 adolescentes) esta población provenía de aquellas zonas de la ciudad con las peores cifras de indicadores sociales en cuanto a: necesidades básicas insatisfechas, pobreza, nivel educativo.

... chiquilines [adolescentes] que tienen el tema de juntadas con chiquilines de la zona, chiquilines más grandes ¿no? Expuestos a muchas situaciones de riesgo. Chiquilines que andan en tiroteos, cosas así viste, expuestos con gente mucho más grande que ellos (...). Y todos de determinados barrios ¿no? [nombra algunos barrios]. (E10-MujerTrabajo Social)

Sumado a los indicadores socioeconómicos que puedan dar cuenta de las características de precariedad de algunos territorios, surge un aspecto extra que tiene que ver con el estigma territorial. Se puede observar cierta exaltación de elementos negativos que se generalizan y se expanden generando consecuencias desacreditadoras para adolescentes o colectivos que de allí provengan. Esta cuestión denominada discriminación estructural (Kessler y Dimarco 2013) provoca fuertes desventajas a la hora de la circulación social en diferentes ámbitos de socialización.

\section{Discusión y Conclusiones}

La presente investigación sigue los enfoques de las etnografías sobre el Estado en la línea que desarrolla Fassin (2015) donde define al Estado como una entidad que es concreta, heterogénea y situada, la cual además puede asirse por sus individualidades o partes que lo integran. Centra la atención en las personas que habitan las instituciones del Estado y lo producen cotidianamente, lejos de ideas abstractas, universales o neutrales. Interesa visibilizar así las relaciones de poder, ideologías y características singulares que adquieren las instituciones estatales en determinados contextos sociales e históricos.

A partir del objetivo de gestionar la privación de libertad de adolescentes se interrelacionan múltiples dispositivos jurídico-burocrácticos de administración estatal (Villalta 2013). Regulaciones, racionalidades y concepciones sobre las formas de ejecución de la medida judicial, donde las prácticas estatales empleadas no son acciones neutrales ni actividades mecanizadas sino que a través del análisis de las mismas podemos visualizar el lado cálido del Estado (Fassin 2015) desde las acciones concretas de las personas que son quienes lo ponen en marcha y dan existencia, así como, desde los diversos significados que le otorgan. Desde esta perspectiva el análisis adquiere sentido solamente en interrelación con el contexto en el que se encuentra.

Situamos además el abordaje feminista sobre el análisis del Estado (Haney 2002) definido como una entidad estratificada en capas, integrado por diversos entes en conflicto que se encuentran débilmente acoplados, en tensión. El Estado construye arquitecturas de necesidad donde se erigen procesos de interpretación de necesidades y redistribución de recursos a partir de definiciones de necesidades, quiénes necesitan y cómo pueden acceder a satisfacerlas. 
En el artículo interesa mostrar el potencial analítico de la noción de cuidados y situarlo como una forma de orientar la ejecución de las medidas socioeducativas en el marco de la privación de libertad. Y también como una alternativa para hacer prevalecer el derecho al cuidado por sobre el avasallamiento de derechos que se producen de los discursos de seguridad y punitivistas sobre la adolescencia y la infracción en Uruguay y la región.

Cabe destacar aquí, al igual que ya lo han demostrado múltiples informes referenciados al inicio, que el encierro, el aislamiento y las condiciones actuales en las que se encuentra la población adolescente en los centros del INISA no constituyen condiciones apropiadas ni dignas para la vida de adolescentes y lo ideal sería tender a la erradicación del encarcelamiento como forma de castigo y planificar la ejecución de estas medidas en otros contextos institucionales o comunitarios con características, recursos humanos y materiales diferentes a los actuales. Dado que sabemos que esta no es una opción a corto plazo porque implicaría un cambio drástico en los paradigmas actuales y de definición política, consideramos como desafío integrar la noción de cuidados, como una cuestión nodal que puede colaborar en el mejoramiento de algunas condiciones y enfoques.

Pensar en la ética de cuidado (Tronto 2013) imperante no implica evaluar el cuidado o valorar prácticas en términos de malas o buenas. Sino que en este ámbito el aporte del estudio tiene que ver con potenciar instancias de reflexión en torno al cuidado de carácter institucional en los centros de privación de libertad y tender a evitar las prácticas discrecionales y dispersas. En este sentido como nombramos al inicio el cuidado aparece como la categoría omitida, algo impensado por algunas personas y reflexionado por otras pero que carece de lugar y definición en la política institucional.

La apuesta de integrar en la ejecución de las medidas privativas de libertad la noción de cuidados es pensar en la posibilidad de reorientar el tratamiento actual que reciben los y las adolescentes desde esta perspectiva y colocando el derecho al cuidado integral en el centro de la gestión. Reconocer así que cuidar es una acción relacional, que implica conflicto y relaciones de poder. Y tender a valorizar las responsabilidades de la sociedad toda en el cuidado y de esta institución puntual de restituir las condiciones de cuidado y garantizar el mismo en sus aspectos materiales y simbólicos.

Por último, la investigación conlleva una serie de limitaciones que merece la pena aclarar. Por una parte, realizar investigación cualitativa en instituciones de encierro comporta múltiples dificultades por el carácter restringido de las mismas y los diversos obstáculos para lograr el permiso de ingreso a la institución. Y posteriormente la permanente negociación para transitar por el espacio, el relacionamiento con la diversidad de agentes institucionales y obtener el acceso a la información en general (Reiter 2014, Myers 2015, Watson y van der Meulen 2018). Por otra, los/as participantes de la investigación han sido seleccionados en función de los objetivos del estudio, sin embargo, sería interesante poder ampliar la muestra utilizada y así abarcar una mayor cantidad de participantes. Así como también incluir para el análisis otros roles y funciones en la institución, como por ejemplo los cargos de definición políticoinstitucional. 


\section{Referencias}

American Psychological Association, 2017. Ethical principles of psychologists and code of conduct [en línea]. Washington, DC. Disponible en:

https://www.apa.org/ethics/code/principles.pdf [Con acceso el 2 de octubre de 2019].

Butler, J., 1993. Cuerpos que importan: Sobre los límites materiales y discursivos del "sexo". Buenos Aires: Paidós.

Butler, J., 2010. Marcos de guerra: Las vidas lloradas. Barcelona: Paidós.

Carrasco, C., Borderías, C., y Torns, T., 2011. Introducción. El trabajo de cuidados: antecedentes históricos y debates actuales. En: C. Carrasco, C. Borderías y T. Torns, eds., El trabajo de cuidados: Historia, teoría y políticas. Madrid: Catarata, 1396.

Comité de los Derechos del Niño Uruguay, 2017. Situación general de los derechos de niñas, niños y adolescentes en Uruguay [en línea]. Informe. Montevideo. Disponible en: http://www.comitednu.org/wp-content/uploads/2014/08/Informe-diagnostico2017.pdf [Con acceso el 2 de octubre de 2019].

Convención sobre los Derechos del Niño, 1989 [en línea]. Nueva York: Asamblea General de las Naciones Unidas, 20 de noviembre. Disponible en: http://www.ohchr.org/SP/ProfessionalInterest/Pages/CRC.aspx [Con acceso el 2 de octubre de 2019].

Crenshaw, K., 2004. Interseccionalidad: una herramienta para la justicia de género y la justicia económica. Derechos de las mujeres y cambio económico [en línea], no 9, 1-10. Disponible en:

https://www.awid.org/sites/default/files/atoms/files/nterseccionalidad una herramienta para la justicia de genero_y la_justicia economica.pdf [Con acceso el 2 de octubre de 2019].

Daly, M., y Lewis, J., 2000. The Concept of Social Care and the Analysis of Contemporary Welfare States. The British Journal of Sociology [en línea], 51(2), 28198. Disponible en: https://doi.org/10.1111/j.1468-4446.2000.00281.x [Con acceso el 2 de octubre de 2019].

Denzin, N., y Lincoln, Y., eds., 2018. The SAGE Handbook of Qualitative Research. 5르. ed. Londres: Sage.

Díaz, D., 2019. La construcción del derecho penal juvenil uruguayo. Montevideo: Fin de Siglo.

Fassin, D., 2015. At the Heart of the State: The Moral World of Institutions [en línea]. Londres: Pluto Press. Disponible en: https://doi.org/10.2307/j.ctt183p5tb [Con acceso el 2 de octubre de 2019].

Fassin, D., 2018a. Castigar: Una pasión contemporánea. Buenos Aires: Adriana Hidalgo.

Fassin, D., 2018b. Por una repolitización del mundo: Las vidas descartables como desafío del siglo XXI. Buenos Aires: Siglo XXI. 
Faur, E., 2014. El cuidado infantil en el siglo XXI: Mujeres malabaristas en una sociedad desigual. Buenos Aires: Siglo XXI.

Fessler, D., 2016. “Menores infractores": Las "nuevas" clases peligrosas en el Uruguay de la inseguridad. En: S.D.M. Freire, ed., Anais do VI Seminário Internacional Direitos Humanos, Violência e Pobreza: A situaçao das crianças e adolescentes na América Latina hoje [en línea]. Río de Janeiro: Rede Sírius / UERJ. Disponible en: http://www.proealc.etc.br/VI SEMINARIO/assets/pdfs/gti/Daniel\%20Fessler\%20 GT01.pdf [Con acceso el 2 de octubre de 2019].

Flyvbejereg, B., 2004. Cinco malentendidos acerca de la investigación mediante los estudios de caso. Reis [en línea], 106(4), 33-62. Disponible en: https://doi.org/10.2307/40184584 [Con acceso el 2 de octubre de 2019].

Foucault, M., 2016. La sociedad punitiva: Curso en el Collége de France (1972-1973). $1^{\mathrm{a}}$ ed. Buenos Aires: Fondo de Cultura Económica.

Gilligan, C., 1982. In a Different Voice: Psychological Theory and Women's Development. Cambridge, MA: Harvard University Press.

Goffman, I., 1963. Estigma: La identidad deteriorada. Buenos Aires: Amorrortu.

Goffman, I., 2001. Internados: Ensayos sobre la situación social de los enfermos mentales. $1^{\text {a }}$ ed., 3를 reimp. Buenos Aires: Amorrortu.

González-Laurino, C., 2015. La infracción adolescente a la ley penal en el centro del debate sobre seguridad pública en Uruguay. Revista latinoamericana de Ciencias Sociales, Niñez y Juventud [en línea], 13(2), 951-62. Disponible en: https://doi.org/10.11600/1692715x.13228201014 [Con acceso el 2 de octubre de 2019].

González-Laurino, C., 2016. Modelos de justicia penal juvenil en debate a inicios del siglo XXI. Quaestio Iuris [en línea], 9(2), 652-69. Disponible en: https://doi.org/10.12957/rqi.2016.18008 [Con acceso el 2 de octubre de 2019].

González-Laurino, C., et al., 2013. Los sentidos del castigo: El debate uruguayo sobre la responsabilidad en la infracción adolescente. Montevideo: Trilce.

González-Laurino, C., y Leopold, S., 2011. Discurso del riesgo y prácticas diagnósticas con niños y adolescentes en el ámbito socio-judicial. Montevideo: Universidad de la República.

González-Laurino, C., y Leopold, S., 2013. De crisis y reformas. El actual funcionamiento del sistema penal juvenil en Uruguay desde la perspectiva de sus actores y expertos. En: C. González Laurino et al., eds., Los sentidos del castigo: El debate uruguayo sobre la responsabilidad en la infracción adolescente. Montevideo: Trilce, pp. 45-69.

González-Laurino, C., y Leopold, S., 2017. La construcción del discurso de la responsabilidad en el sistema penal Juvenil. En: R. Abella y D. Fessler, eds., El retorno del "estado peligroso": Los vaivenes del sistema penal juvenil. Montevideo: Casa Bertolt Brecht / Grupo de Estudios sobre Infracción Adolescente, CSICUDELAR. 
Guber, R., 2013. La articulación etnográfica: Descubrimiento y trabajo de campo en la investigación de Esther Hermitte. Buenos Aires: Biblos.

Hammersley, M., y Atkinson, P., 1994. Etnografía: Métodos de investigación. Barcelona: Paidós.

Haney, L., 2002. Inventing the Needy: Gender and the Politics of Welfare in Hungary. Berkeley / Los Angeles: University of California Press.

Haney, L., 2004. Introduction: Gender, Welfare, and States of Punishment. Social Politics [en línea], 11(3), 333-62. Disponible en: https://doi.org/10.1093/sp/jxh040 [Con acceso el 2 de octubre de 2019].

Haraway, D., 1995. Ciencia, cyborgs y mujeres: La reinvención de la naturaleza. Madrid: Cátedra.

Harding, S.G., 2006. Ciencia y feminismo. Madrid: Morata.

Hochschild, A., 2011. La mercantilización de la vida íntima: Apuntes de la casa y el trabajo. Madrid: Katz.

Institución Nacional de Derechos Humanos y Defensoría del Pueblo, 2017. III Informe Anual del Mecanismo Nacional de Prevención de la Tortura de la República Oriental del Uruguay 2016: El MNP en el Uruguay: puesta en funcionamiento y consolidación [en línea]. Montevideo. Disponible en:

https://www.ohchr.org/Documents/HRBodies/OPCAT/NPM/NMPUruguay2016. pdf [Con acceso el 2 de octubre de 2019].

Institución Nacional de Derechos Humanos y Defensoría del Pueblo, 2018. IV Informe Anual del Mecanismo Nacional de Prevención de la Tortura: Uruguay 2017 [en línea]. Montevideo. Disponible en: https://www.gub.uy/institucion-nacional-derechoshumanos-uruguay/sites/institucion-nacional-derechos-humanosuruguay/files/documentos/publicaciones/1-INDDHH digital.pdf [Con acceso el 2 de octubre de 2019].

Institución Nacional de Derechos Humanos y Defensoría del Pueblo, 2019. V Informe Anual del Mecanismo Nacional de Prevención de la Tortura: Uruguay 2018 [en línea]. Montevideo. Disponible en: https://www.gub.uy/institucion-nacional-derechoshumanos-uruguay/comunicacion/publicaciones/informe-2018-del-mecanismonacional-prevencion-tortura-mnp [Con acceso el 2 de octubre de 2019].

Kessler, G., y Dimarco, S., 2013. Jóvenes, policía y estigmatización territorial en la periferia de Buenos Aires. Espacio Abierto Cuaderno Venezolano de Sociología [en línea], 22(2), 221-43. Disponible en:

https://www.redalyc.org/pdf/122/12226914003.pdf [Con acceso el 2 de octubre de 2019].

Leopold, S., 2014. Los laberintos de la infancia: Discursos, representaciones y crítica. Montevideo: Ediciones Universitarias.

López-Gallego, L., 2014. Proceso de reflexividad en un contexto de privación de libertad de adolescentes mujeres. Psicologia \& Sociedade [en línea], 26(3), 603-612. Disponible en: https://doi.org/10.1590/S0102-71822014000300009 [Con acceso el 2 de octubre de 2019]. 
López-Gallego, L., 2017 Procesos de constitución del sistema penal juvenil uruguayo. ¿Paradigmas híbridos? Crítica Penal y Poder [en línea], no 12, pp. 109-125.

Disponible en: https://www.raco.cat/index.php/CPyP/article/view/321884 [Con acceso el 2 de octubre de 2019].

López-Gallego, L., y Padilla, A., 2013. Responsabilidad adolescente y prácticas psi: Relaciones peligrosas. En: C. González Laurino et al., eds., Los sentidos del castigo: El debate uruguayo sobre la responsabilidad en la infracción adolescente. Montevideo: Trilce, pp. 71-94.

Medan, M., 2017. Prevención social y delito juvenil. El gobierno de la juventud en riesgo en el AMBA: entre la seguridad y la inclusión [en línea]. Universidad de Buenos Aires. Disponible en: https://www.teseopress.com/elgobiernodelajuventudenriesgo [Con acceso el 2 de octubre de 2019].

Merklen, D., 2013. Las dinámicas contemporáneas de la individuación. En: R. Castel et al., eds., Individuación, precariedad, inseguridad: ¿desinstitucionalización del presente? Buenos Aires: Paidós, 45-86.

Montes-Maldonado, C., 2019. Sentidos del cuidado en centros de privación de libertad para adolescentes en Uruguay. Revista Latinoamericana de Ciencias Sociales, Niñez y Juventud [en línea], 17(2), pp. 1-22. Disponible en: https://doi.org/10.11600/1692715x.17216 [Con acceso el 2 de octubre de 2019].

Morás, L.E., 2012. Los hijos del estado (vol. 1). Montevideo: SERPAJ.

Morás, L.E., 2016. Estudio de las trayectorias de vida de adolescentes en conflicto con la ley con particular énfasis en la relación delito-trabajo [en línea]. Informe final. Montevideo: OIT / Cinterfor. Disponible en: http://www.oitcinterfor.org/sites/default/files/file publicacion/moras web.pdf [Con acceso el 2 de octubre de 2019].

Myers, R., 2015. Barriers, Blinders, and Unbeknownst Experts: Overcoming Access Barriers to Conduct Qualitative Studies of Juvenile Justice. The Prison Journal [en línea], 95(1), 66-83. Disponible en: https://doi.org/10.1177/0032885514563279 [Con acceso el 2 de octubre de 2019].

Pautassi, L., 2010. Cuidado y derechos: la nueva cuestión social. En: S. Montaño y C. Calderón, eds., El cuidado en acción: entre el derecho y el trabajo. Santiago de Chile: Comisión Económica para América Latina y el Caribe (CEPAL), 69-92.

Reiter, K., 2014. Making Windows in Walls: Strategies for Prison Research. Qualitative Inquiry [en línea], 20(4), 417-428. Disponible en:

https://doi.org/10.1177/1077800413515831 [Con acceso el 2 de octubre de 2019].

Rodríguez-Alzueta, E., ed., 2016. Hacer bardo: Provocaciones, resistencias y derivas de jóvenes urbanos. $1^{\underline{a}}$ ed. La Plata: Malisia.

Ruiz Barbot, M., et al., 2018. Consulta a adolescentes privados de libertad. Uruguay, 2018 [en línea]. Montevideo: UNICEF. Disponible en: http://radiouruguay.uy/wpcontent/uploads/2019/01/Consulta-a-adolescentes-privados-de-libertad.pdf [Con acceso el 2 de octubre de 2019]. 
Ruiz Olabuénaga, J.I., 2012. Metodología de la investigación cualitativa. Bilbao: Universidad de Deusto.

Spencer, L., et al., 2014. Analysis in Practice. En: J. Ritchie et al., eds., Qualitative Research Practice: A Guide for Social Science Students and Researchers. $2^{a}$ ed. Londres: Sage, 295-345.

Tronto, J., 1993. Moral Boundaries: A Political Argument for an Ethic of Care. Nueva York: Routledge.

Tronto, J., 2005. Cuando la ciudadanía se cuida: una paradoja neoliberal del bienestar y la desigualdad. En: Emakunde/Instituto Vasco de la Mujer, ed., Congreso Internacional Sare 2004: ¿Hacia qué modelo de ciudadanía? [en línea]. Vitoria-Gasteiz: Emakunde/Instituto Vasco de la Mujer, 231-53. Disponible en: https://www.emakunde.euskadi.eus/contenidos/informacion/publicaciones jorna das/es emakunde/adjuntos/sare2004 es.pdf [Con acceso el 2 de octubre de 2019].

Tronto, J., 2013. Caring Democracy: Markets, Equality, and Justice. New York University Press.

Uriarte, C., 2013. La cuestión de la responsabilidad en el derecho penal juvenil. En: C. González-Laurino et al., eds., Los sentidos del castigo: El debate uruguayo sobre la responsabilidad en la infracción adolescente. Montevideo: Trilce, 141-59.

Vianna, A., 2010. Derechos, moralidades y desigualdades: consideraciones a partir de procesos de guarda de niños. En: C. Villalta, ed., Infancia, justicia y derechos humanos. Bernal: Editorial de la Universidad Nacional de Quilmes.

Villalta, C., 2013. Un campo de investigación: las técnicas de gestión y los dispositivos jurídico-burocráticos destinados a la infancia pobre en la Argentina. Civitas [en línea], 13(2), pp. 245-268. Disponible en: http://doi.org/10.15448/19847289.2013.2.15482 [Con acceso el 2 de octubre de 2019].

Wacquant, L., 2012. Castigar a los pobres: El gobierno neoliberal de la inseguridad social. Barcelona: Gedisa.

Watson, T.M., y van der Meulen, E., 2018. Research in carceral contexts: confronting access barriers and engaging former prisoners. Qualitative Research [en línea], 19(2), 182-198. Disponible en: https://doi.org/10.1177/1468794117753353 [Con acceso el 2 de octubre de 2019].

Yin, R.K., 1993. Applications of Case Study Research. Londres: Sage.

\section{Referencias normativas}

Ley № 16.137. Aprobación de la Convención sobre los derechos del niño. Registro Nacional de Leyes y Decretos [en línea], tomo 1, semestre 2, p. 365. Disponible en: http://www.impo.com.uy/bases/leyes/16137-1990 [Con acceso el 2 de octubre de 2019].

Ley № 17.823, Código de la Niñez y Adolescencia. D.O. [en línea], nº 26586, de 14 de septiembre de 2004. Disponible en:

https://www.oas.org/dil/esp/Codigo Ninez Adolescencia Uruguay.pdf [Con acceso el 2 de octubre de 2019]. 
Ley № 19.055. Código de la niñez y la adolescencia. Se modifican los artículos 72 y 73 y se establece un régimen especial para adolescentes mayores de quince y menores de dieciocho. D.O. [en línea], no 28642, de 22 de enero de 2013. Disponible en: https://legislativo.parlamento.gub.uy/temporales/leytemp4440326.htm [Con acceso el 2 de octubre de 2019].

Ley № 19.367. Creación del Instituto Nacional de Inclusión Social Adolescente como servicio descentralizado. D.O. [en línea], nº 29384, de 27 de enero de 2016. Disponible en:

https://legislativo.parlamento.gub.uy/temporales/leytemp965450.htm [Con acceso el 2 de octubre de 2019]. 